\title{
Risk management to prioritise the eradication of new and emerging invasive non-native species
}

\author{
Olaf Booy $\cdot$ Aileen C. Mill $\cdot$ Helen E. Roy $\cdot$ Alice Hiley $\cdot$ Niall Moore $\cdot$ \\ Pete Robertson - Simon Baker • Matt Brazier • Mathilde Bue • Richard Bullock • \\ Steve Campbell $\cdot$ Dominic Eyre $\cdot$ Jim Foster $\cdot$ Maggie Hatton-Ellis • \\ Jo Long • Craig Macadam • Camilla Morrison-Bell • John Mumford • \\ Jonathan Newman - David Parrott • Robin Payne - Trevor Renals • \\ Eoina Rodgers $\cdot$ Mark Spencer $\cdot$ Paul Stebbing $\cdot$ Mike Sutton-Croft • \\ Kevin J. Walker · Alastair Ward $\cdot$ Stan Whittaker $\cdot$ Gabe Wyn
}

Received: 17 November 2016/ Accepted: 28 April 2017/Published online: 15 May 2017

(C) The Author(s) 2017. This article is an open access publication

\begin{abstract}
Robust tools are needed to prioritise the management of invasive non-native species (INNS). Risk assessment is commonly used to prioritise INNS, but fails to take into account the feasibility of management. Risk management provides a structured evaluation of management options, but has received little attention to date. We present a risk management scheme to assess the feasibility of eradicating INNS that can be used, in conjunction with existing risk
\end{abstract}

Electronic supplementary material The online version of this article (doi:10.1007/s10530-017-1451-z) contains supplementary material, which is available to authorized users.

O. Booy · N. Moore $\cdot$ P. Robertson - S. Baker ·

D. Parrott $\cdot$ M. Sutton-Croft $\cdot$ A. Ward

National Wildlife Management Centre, Animal and Plant

Health Agency, Sand Hutton, York YO41 1LZ, UK

O. Booy $(\bowtie) \cdot$ A. C. Mill · P. Robertson

Centre for Wildlife Management, School of Biology,

Newcastle University, Newcastle-upon-Tyne NE1 7RU, UK

e-mail: olaf.booy@apha.gsi.gov.uk

H. E. Roy · J. Newman

Centre for Ecology and Hydrology, Maclean Building,

Benson Lane, Crowmarsh Gifford, Wallingford,

Oxfordshire OX10 8BB, UK

A. Hiley · M. Brazier · T. Renals

Environment Agency, Tyneside House, Skinnerburn

Road, Newcastle upon Tyne NE4 7AR, UK assessment schemes, to support prioritisation. The Non-Native Risk Management scheme (NNRM) can be applied to any predefined area and any taxa. It uses semi-quantitative response and confidence scores to assess seven key criteria: Effectiveness, Practicality, Cost, Impact, Acceptability, Window of opportunity and Likelihood of re-invasion. Scores are elicited using expert judgement, supported by available evidence, and consensus-building methods. We applied the NNRM to forty-one INNS that threaten Great Britain (GB). Thirty-three experts provided scores, with overall feasibility of eradication assessed as 'very high' (8 species), 'high' (6), 'medium' (8), 'low' (10)

\author{
M. Bue \\ Institute of Biological, Environmental and Rural Sciences, \\ Aberystwyth University, Aberystwyth SY23 3DA, UK \\ R. Bullock \\ Wildfowl and Wetlands Trust, Queen Elizabeth's Walk, \\ Barnes, London SW13 9WT, UK \\ S. Campbell \\ Science and Advice for Scottish Agriculture, Roddinglaw \\ Road, Edinburgh EH12 9FJ, UK \\ D. Eyre \\ Defra, Sand Hutton, York YO41 1LZ, UK \\ J. Foster \\ Amphibian and Reptile Conservation, The Witley Centre, \\ Witley, Godalming, Surrey GU8 5QA, UK
}


and 'very low' (9). The feasibility of eradicating terrestrial species was higher than aquatic species. Lotic freshwater and marine species scored particularly low. Combining risk management and existing risk assessment scores identified six established species as priorities for eradication. A further six species that are not yet established were identified as priorities for eradication on arrival as part of contingency planning. The NNRM is one of the first INNS risk management schemes that can be used with existing risk assessments to prioritise INNS eradication in any area.

Keywords Risk analysis - Resource management . Feasibility of eradication - Contingency planning . Great Britain · NNRM

\section{Introduction}

Decision-makers are under growing pressure to respond to invasive non-native species (INNS) (Hulme 2006; Seebens et al. 2017) and require transparent evidence on which to base decisions (Sutherland et al. 2004). Given the large and increasing numbers of species involved (Pimentel et al. 2005; Daisie 2009; Hulme 2009; Roy et al. 2014a), the often high costs associated with management and limited resources, there is a need for management to be

M. Hatton-Ellis · G. Wyn

Natural Resources Wales, Maes y Ffynnon, Penrhos Rd,

Bangor LL572DW, UK

C. Morrison-Bell

British Ecological Society, Charles Darwin House, 12

Roger Street, London WC1N 2JU, UK

\section{J. Long}

Scottish Environment Protection Agency, Strathallan

House, Castle Business Park, Stirling FK9 4TZ, UK

C. Macadam

Buglife, Balallan House, 24 Allan Park,

Stirling FK8 2QG, UK

\section{J. Mumford}

Imperial College London, Silwood Park Campus, Ascot, Berkshire SL5 7PY, UK

R. Payne

Airlie Bank, Bamff Road, Alyth PH11 8DR, Scotland, UK carefully prioritised (McGeoch et al. 2016; Early et al. 2016). However, practical, transparent and robust tools to support the prioritisation of management are lacking (Hulme et al. 2009).

One way of prioritising INNS management is to use risk analysis, which traditionally includes hazard identification, risk assessment, risk management and risk communication (Vanderhoeven et al. 2017). It is the balance between risk assessment and risk management that allows for prioritisation, with risk assessment used to assess the threat or hazard of a species and risk management used to evaluate and implement management options (FAO 1995). Within this framework, high risk species for which management is cost effective are prioritised first and low risk species for which management is expensive and ineffective are prioritised last. Both risk assessment and risk management are essential for prioritisation; however, while numerous INNS risk assessment schemes have been developed (for reviews see Early et al. 2016; Heikkilä 2011; Leung et al. 2012; Roy et al. 2014b; Verbrugge et al. 2010) very few exist for risk management (Heikkilä 2011; Vanderhoeven et al. 2017). Of the schemes that do include elements of risk management, many only include one or few questions (e.g. Essl et al. 2011; Vanderhoeven et al. 2015) or provide an evaluation of what is advisable, but not an indication of priority (Schmiedel et al. 2016). While more elaborate schemes are available for weed risk management and plant health pests, these are limited

\section{E. Rodgers}

Royal Society for the Prevention of Cruelty to Animals, Wilberforce Way, Southwater RH13 9RS, UK

M. Spencer

Natural History Museum, Cromwell Road,

London SW7 5BD, UK

P. Stebbing

Centre for Environment, Fisheries and Aquaculture

Science, The Nothe, Barrack Road, Weymouth,

Dorset DT4 8UB, UK

\section{K. J. Walker}

Botanical Society of Britain and Ireland, Natural History Museum, Cromwell Road, London SW7 5BD, UK

S. Whittaker

Scottish Natural Heritage, Silvan House, Corstorphine Road, Edinburgh EH12 7AT, UK 
by being taxonomically or sector specific (e.g. Auld 2012; Baker et al. 2005; Drolet et al. 2014; Firn et al. 2015a, b; Hiebert and Stubbendieck 1993; Kehlenbeck et al. 2012; Setterfield et al. 2010; Sunley et al. 2012; Virtue 2010), consider only specific aspects of risk management (e.g. Darin 2008; Hauser and McCarthy 2009; Darin et al. 2011; Liu et al. 2011) or being time and resource intensive (e.g. Darin et al. 2011; Leung et al. 2012; McGeoch et al. 2016; Vander Zanden et al. 2010).

There is, therefore, a need for a practical risk management scheme that is compatible with existing risk assessment schemes in order to support prioritisation of INNS (D'hondt et al. 2015). Given the range of species that become invasive, such a scheme should be broadly applicable to any taxa (Nentwig et al. 2010) and, given large numbers of species involved, should be efficient to apply (Andersen et al. 2004; Hulme et al. 2009). It should be possible to complete the scheme even where data are lacking, with uncertainty taken into account, documented and justified (Leung et al. 2012; Vanderhoeven et al. 2017).

We set out to develop a scheme, known as the NonNative Risk Management Scheme (NNRM), that meets these criteria and complies with international standards for risk management (FAO 1995, 2006; OIE 2015) as well as good practice for prioritisation (summarised by Heikkilä 2011). We focussed on assessing the feasibility of eradication (sensu Genovesi 2000), acknowledging that eradication is the most effective management response after prevention (Genovesi 2005). It is also the focus of the second tier in the hierarchical approach to INNS management (Guiding Principle 2, COP 6 decision VI/23, Convention on Biological Diversity) as well as an important component of Aichi Target 9 (UNEP 2011). To ensure it could be practically applied and completed even where data were limited, the NNRM was designed to use expert judgement (Martin et al. 2012) to provide semi-quantitative scores (sensu Baker et al. 2008) which are justified by written comments, and supported by evidence where available. This follows similar approaches used for risk assessment (Baker et al. 2008, 2012; Essl et al. 2011; Leung et al. 2012).

To demonstrate its use we trialled the scheme in $\mathrm{GB}$, which has a well-developed and robust INNS risk assessment process but lacks a compatible process for risk management (Defra 2015). We applied the scheme to a group of new and emerging INNS that pose a threat to GB, as these were considered most likely to be potential candidates for eradication (Roy et al. 2014c). We demonstrate how the scheme can be used, in combination with existing risk assessment scores, to indicate priorities for eradication and contingency planning; and examine the importance of risk management for prioritisation. While applied here to GB, the scheme can be applied at different scales and in different areas worldwide. Indeed, the scheme may have particular application in the EU where the recent adoption of Regulation No. 1143/2014 includes requirements for eradication of INNS listed on the basis of both risk assessment and risk management variables (EU 2014).

\section{Materials and methods}

\section{Development}

The Non-Native Risk Management Scheme (NNRM) was developed over a 2-year period from 2013 to 2015 in collaboration with INNS management and risk analysis experts from Great Britain (GB). Initial criteria were developed in consultation with this group taking into consideration existing literature on INNS risk analysis and eradication (in particular Baker et al. 2005; Cacho et al. 2006; Cunningham et al. 2003; Genovesi 2005, 2007; Hiebert and Stubbendieck 1993; Hulme 2006; Johnson 2009; Kehlenbeck et al. 2012; Mehta et al. 2010; Randall et al. 2008; Rejmánek and Pitcairn 2002; Simberloff 2003, 2009; Sunley et al. 2012; Virtue 2010). Refinements were made to the scheme during an initial trial in March 2014 and subsequently the expert elicitation and consensus-building process described below. Decision-makers were engaged in the initial development of the scheme and at intervals throughout the process to ensure the relevance of the scheme for them as endusers.

The non-native risk management scheme

The NNRM takes the form of a questionnaire supported by guidance (S1), which is summarised in Box 1. Preliminary stages record the details of authors, the organism to be assessed, the risk management area and the objective of the assessment. The risk management area is user defined to allow any area 
to be assessed, but must be precisely defined. The objective of the assessment is set from the outset as the complete eradication of the organism from the risk management area (sensu Genovesi 2000).

Once preliminary stages are complete, the assessment is started by documenting the invasion scenario (Box 1, step 1), which describes the extent of the INNS in the risk management area (see guidance in S1 for detail). The scenario may be based on known existing or predicted future invasions, as well as probabilistic scenarios such as best, most likely, or worst case scenarios; however, for assessments to be comparable the scenario selected must be consistent (to this end the most likely scenario was adopted for all assessments in the trial described below). Multiple scenarios may be considered for individual species, in which case each scenario is assessed separately. In all cases assessors should carefully document the scenario being considered, along with any assumptions made, to provide context for the results.
The eradication strategy is then defined (Box 1, step 2). This is a realistic strategy considered likely to achieve complete eradication of the species from the defined risk management area and can include any combination of individual methods (e.g. use of pesticides, herbicides, trapping, shooting, etc.). Multiple eradication strategies can be considered if necessary to allow for comparison between different approaches, in which case each strategy should be separately assessed. Assessors determine which strategy they consider likely to achieve eradication, avoiding being too conservative (i.e. no eradication possible despite techniques being available) or unrealistic (i.e. cost/damage caused vastly outweighs potential benefits). If no realistic eradication strategy can be determined then the species automatically scores very low overall feasibility of eradication and comments are provided to justify this decision. As with the invasion scenario, defining the eradication strategy at this point allows for assumptions to be

Box 1 Summary of guidance provided to complete risk management assessments; the full scheme is available (S1)

1. Define the invasion scenario. For species that are already established this is the current extent of the species in the risk management area. For species on the horizon this is the most likely extent of the species in the risk management area at the point detection could reasonably be expected (based on existing surveillance)

2. Define the eradication strategy. Based on the defined scenario briefly describe the eradication strategy being assessed. This should be a realistic strategy you consider most likely to be effective in eradicating the species completely from the risk management area. The overall strategy could include multiple methods (e.g. use of pesticides, herbicides, trapping, shooting, etc.) and should include any other work that would be required such as surveys, logistics and monitoring

3. Assess the eradication strategy:

(a) Effectiveness. How effective would the eradication strategy be? This relates to how effective the defined strategy would be if it could be deployed regardless of issues with practicality, cost, impact and acceptability

(b) Practicality. How practical would it be to deliver the eradication strategy? This includes issues such as gaining access to relevant areas, obtaining appropriate equipment, skilled staff or pesticides. If there are any legal barriers to undertaking the work these are assessed here

(c) Cost. How much would the eradication strategy cost? This is the total direct cost of the strategy including materials, staff time and any other direct costs. Indirect costs, such as loss of business, are taken into account under negative impact (3d)

(d) Negative impact. What negative impact would the eradication strategy have? Assess the impact that the eradication strategy itself would have on the environment, economy or society

(e) Acceptability. How acceptable is the eradication strategy? Could the eradication strategy meet significant disapproval or resistance from the general public, key sectors or any other stakeholder?

4. Assess the window of opportunity for delivering the described eradication strategy. How quickly will the species spread beyond the point that eradication, using the defined strategy, would be effective?

5. Assess the likelihood of re-invasion following eradication. Unless the eradication strategy deliberately targets populations in containment or otherwise not in the wild (i.e. in gardens, zoos, etc.) introduction from these sources should be considered potential sources of re-invasion. If relevant, the eradication strategy could include pathway management measures in order to reduce this score

6. Overall feasibility of eradication. Taking into account all preceding scores, provide an overall score for the feasibility of eradicating this species from the risk management area

The risk management objective was set as complete eradication from GB for all species 
documented and a clear basis for the rest of the assessment to be set.

The feasibility of eradication, based on the defined eradication strategy, is then assessed using seven key questions relating to Effectiveness, Practicality, Cost, Impact, Acceptability, Window of opportunity and Likelihood of reinvasion (Box 1, steps 3a-e, 4 and 5). Lastly, the assessor provides a single overall score for the feasibility of eradication (Box 1, step 6), which is based on their expert judgement taking account of the scenario and responses made in the previous steps. The overall score is not directly calculated from individual scores, because no appropriate weighting could be identified that would account for the wide range of taxa and criteria being assessed (Mumford et al. 2010). Instead we used expert judgement based on previous steps, which follows the approach used by the UK, EPPO and other risk assessment schemes (Baker et al. 2012; Mumford et al. 2010) and provides flexibility, while ensuring overall scores are supported by individual scores and documented justification.

\section{Response and confidence scores}

For each of the seven questions and the overall conclusion a response and confidence score are required with justification provided by a written comment. Response scores are ordinal on a five-point scale with one being least favourable and five being most (Table 1). Each alternative response is predefined using descriptive terms (similar to those used in risk assessment schemes, e.g. Baker et al. 2008, 2012), except for Cost and Window of opportunity which is based on quantified bands. Bands for Cost scores were determined in consultation with decision makers that hold national budgets for INNS control and reflect the range of costs associated with historical eradication attempts that have been made in GB (if applied to other countries/regions these bands may need to be recalibrated). Window of opportunity was quantified in consultation with risk management experts to reflect timescales likely to be relevant to a wide range of taxa. Confidence scores are explicitly recorded for every response using a three point scale (low, medium high) following Mumford et al. (2010), which in turn is based on a simplification of guidance provided by the Intergovernmental Panel on Climate Change (Mastrandrea et al. 2011).
Applying the scheme to new and emerging threats to GB

The scheme was used to assess 41 new or emerging INNS that pose a threat to GB and represent a broad range of taxa and environments (Tables 2, 3a, b). Twenty species were already established in GB at the time of assessment, but with limited distributions; a further 21 were horizon species, defined as species not established in GB at the time of assessment but considered likely to invade in the near future. The list of horizon species was based on the top 30 threats identified by Roy et al. (2014c), less nine species that were excluded. Species were excluded if they were primarily crop, forestry or fish pests and dealt with by established plant or fish health regimes in GB; or were species that had already established in GB by the time of assessment, in which case they were included as established species. The remaining established species were selected based on their limited distributions in GB and because they were being considered for potential eradication by national policy makers in GB. The most likely scenario was used for all species, which for established species was defined as the species' current extent, and for horizon species was the most likely extent at the point of detection with existing surveillance.

We used expert judgement (supported by evidence where available) to elicit scores, which is practical but must be used carefully to minimise the impacts of subjectivity, bias and group think (Burgman et al. 2011; Martin et al. 2012; Sutherland and Burgman 2015). To this end we followed the approach used by Roy et al. (2014c) which combines expert elicitation with review and consensus building to reduce these effects, while still being practical and efficient to apply. Techniques incorporated within this approach include: (a) the structured use of groups rather than individuals to produce scores, (b) independent initial scoring followed by review and consensus building; (c) transparent, documented justification of all scores; (d) initial presentations and discussion around the scoring method and terminology to reduce the potential for language based misunderstanding; (e) open facilitator-led discussions to encourage all participants to listen to one another, asses each other's judgements and cross examine reasoning behind scores; (f) breakout sessions to provide smaller and more informal space in which to express views; and (g) agreeing final 
Table 1 Assessment criteria for response scores, 1 is least favourable and 5 the most

\begin{tabular}{|c|c|c|c|c|c|}
\hline \multirow[t]{2}{*}{ Criteria } & \multicolumn{5}{|l|}{ Response score } \\
\hline & 1 & 2 & 3 & 4 & 5 \\
\hline Effectiveness & Very ineffective & Ineffective & $\begin{array}{l}\text { Moderate } \\
\text { effectiveness }\end{array}$ & Effective & Very effective \\
\hline Practicality & Very impractical & Impractical & $\begin{array}{l}\text { Moderate } \\
\text { practicality }\end{array}$ & Practical & Very practical \\
\hline Cost & $>£ 10 \mathrm{M}$ & $£ 1-10 \mathrm{M}$ & $£ 200 \mathrm{k}-1 \mathrm{M}$ & $£ 50-200 \mathrm{k}$ & $<£ 50 \mathrm{k}$ \\
\hline Negative impact & Massive & Major & Moderate & Minor & Minimal \\
\hline Acceptability & $\begin{array}{l}\text { Very } \\
\text { unacceptable }\end{array}$ & Unacceptable & $\begin{array}{l}\text { Moderate } \\
\text { acceptability }\end{array}$ & Acceptable & $\begin{array}{l}\text { Very } \\
\text { acceptable }\end{array}$ \\
\hline Window of opportunity & $<2$ months & $\begin{array}{l}2 \text { months- } \\
1 \text { year }\end{array}$ & $1-3$ years & $4-10$ years & $>10$ years \\
\hline Likelihood of reinvasion & Very likely & Likely & Moderate likelihood & Unlikely & Very unlikely \\
\hline $\begin{array}{l}\text { Conclusion (overall feasibility of } \\
\text { eradication) }\end{array}$ & Very low & Low & Medium & High & Very high \\
\hline
\end{tabular}

Table 2 Establishment status and environment of species used to test the risk management scheme

\begin{tabular}{|c|c|c|c|c|c|}
\hline \multirow[t]{2}{*}{ Taxa } & \multicolumn{3}{|c|}{ Environment } & \multicolumn{2}{|l|}{ Status in GB } \\
\hline & Terrestrial & Freshwater & Marine & Established & Not established \\
\hline Plants & 5 & 5 & 1 & 8 & 3 \\
\hline Vertebrates & 10 & 3 & 0 & 6 & 7 \\
\hline Invertebrates & 2 & 8 & 7 & 8 & 9 \\
\hline Totals & 17 & 16 & 8 & 22 & 19 \\
\hline
\end{tabular}

Species were selected using the list of the top 30 horizon scanning species for Great Britain (Roy et al. 2014c) as well as species with limited distribution currently being considered for eradication by national policy makers in GB

scores through a facilitator-led discussion where every participant was directly invited to comment on each score. We did not attempt to weight individual expert judgements because of practical problems associated with constructing reliable and valid weights (Bolger and Rowe 2015).

In total, 33 experts were engaged in the elicitation process divided into four groups comprising 7-10 experts each: freshwater animals; terrestrial animals; marine species; and plants, excluding marine plants. Experts were selected based on their proven experience of INNS management in GB and diversity of background (i.e. government, non-government, practitioners, academics and policy advisors). Experts were provided with guidance (S1) and instructions to carry out their assessments. They were encouraged to discuss any points of clarification either with their group leader or the organisers. Clarification of any points was circulated to all experts for consistency. Initial risk management assessments were drafted over a period of 7 weeks by experts from each group using published or grey literature to support scores and expert judgement where other forms of evidence were lacking or inconclusive. The task of completing assessments was shared between experts, with each species being assessed by a single expert. Drafted assessments were then circulated to other experts in the group to provide an initial opportunity for review and comment before the consensus building workshop.

The consensus building workshop took place on 28 April 2015 and attended by 19 of the original experts (limited due to availability), with the drafted risk management assessments used as the basis of the 
Table 3 Risk management scores

\begin{tabular}{|c|c|c|c|c|c|c|c|c|c|c|c|c|c|}
\hline \multirow[b]{2}{*}{ Species } & \multirow[b]{2}{*}{$\begin{array}{l}\text { Invasion scenario } \\
\text { (brief summary) }\end{array}$} & \multirow[b]{2}{*}{$\begin{array}{l}\text { Eradication strategy } \\
\text { (brief summary) }\end{array}$} & \multirow[b]{2}{*}{ 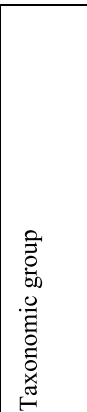 } & \multirow[b]{2}{*}{ 壱 } & \multicolumn{9}{|c|}{ Risk management } \\
\hline & & & & & 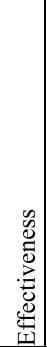 & 穿 & 悹 & $\begin{array}{l}\tilde{\Xi} \\
\tilde{\Xi} \\
\tilde{\Xi}\end{array}$ & 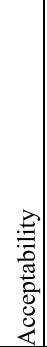 & 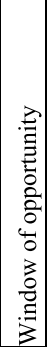 & 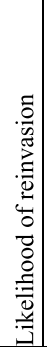 & 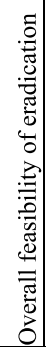 & 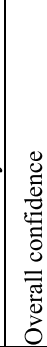 \\
\hline \multicolumn{14}{|c|}{ (a) Species already established in GB, but with limited distribution } \\
\hline $\begin{array}{l}\text { Baccharis halimifolia } \\
\text { (Sea Myrtle) }\end{array}$ & 1 site on south coast of England & $\begin{array}{l}\text { Hand removal and cut stump } \\
\text { treatment with glyphosate }\end{array}$ & Plant & $\mathrm{T}$ & 5 & 5 & 5 & 4 & 4 & 5 & 4 & 5 & $\mathrm{H}$ \\
\hline $\begin{array}{l}\text { Procambarus acutus } \\
\text { (White River Crayfish) }\end{array}$ & 1 site, $<4$ angling ponds (lentic) & $\begin{array}{l}\text { Remove specimen fish and } \\
\text { treat with biocides }\end{array}$ & Invert. & $\mathrm{F}$ & 4 & 5 & 4 & 5 & 4 & 3 & 4 & 5 & $\mathrm{H}$ \\
\hline $\begin{array}{l}\text { Zamenis longissimus } \\
\text { (Aesculapian Snake) }\end{array}$ & 2 sites, each c. $<100$ individuals & $\begin{array}{l}\text { Intensive capture and habitat } \\
\text { manipulation }\end{array}$ & Rept. & $\mathrm{T}$ & 5 & 5 & 4 & 5 & 3 & 4 & 4 & 5 & $\mathrm{H}$ \\
\hline $\begin{array}{l}\text { Sarracenia purpurea } \\
\text { (Purple Pitcher-plant) }\end{array}$ & $\begin{array}{l}14 \text { populations in total covering } \\
<10 \text { hectares }\end{array}$ & $\begin{array}{l}\text { Combination of hand pulling } \\
\text { and herbicidal treatment }\end{array}$ & Plant & $\mathrm{T}$ & 4 & 4 & 4 & 4 & 5 & 5 & 3 & 4 & $\mathrm{H}$ \\
\hline $\begin{array}{l}\text { Lacerta bilineata } \\
\text { (Green Lizard) }\end{array}$ & $\begin{array}{l}1 \text { population }(<1000 \\
\text { individuals) in cliff terrain }\end{array}$ & $\begin{array}{l}\text { Intensive capture and habitat } \\
\text { manipulation }\end{array}$ & Rept. & $\mathrm{T}$ & 4 & 3 & 3 & 4 & 4 & 4 & 3 & 4 & $\mathrm{H}$ \\
\hline $\begin{array}{l}\text { Orconectes limosus } \\
\text { (Spiny-cheek Crayfish) }\end{array}$ & 3 pond and lake sites (lentic) & $\begin{array}{l}\text { Biocides (as described by Peay } \\
\text { et al, 2006) }\end{array}$ & Invert. & $\mathrm{F}$ & 4 & 4 & 3 & 3 & 3 & 4 & 4 & 4 & $\mathrm{M}$ \\
\hline $\begin{array}{l}\text { Cabomba caroliniana } \\
\text { (Fanwort) }\end{array}$ & $\begin{array}{l}1 \text { population (many colonies) in } \\
\text { Basingstoke Canal (SSSI), total } \\
\text { area c. } 800 \mathrm{~m}^{2} \text { (lotic) }\end{array}$ & $\begin{array}{l}\text { Repeated mechanical control } \\
\text { (no herbicides available) }\end{array}$ & Plant & $\mathrm{F}$ & 5 & 4 & 5 & 2 & 2 & 5 & 3 & 3 & $\mathrm{M}$ \\
\hline $\begin{array}{l}\text { Hydropotes inermis } \\
\text { (Chinese Water Deer) }\end{array}$ & $\begin{array}{l}2000+\text { individuals; core } \\
\text { population in East Anglia, } \\
\text { scattered elsewhere }\end{array}$ & Trapping and shooting & Mam. & $\mathrm{T}$ & 5 & 3 & 2 & 5 & 3 & 4 & 4 & 3 & $\mathrm{M}$ \\
\hline $\begin{array}{l}\text { Aponogeton distachyos } \\
\text { (Cape Pondweed) }\end{array}$ & $\begin{array}{l}\text { c. } 80 \text { scattered populations; } \\
\text { well established in c. } 75 \% \text {, } \\
\text { primarily in lakes and ponds } \\
\text { (lentic) }\end{array}$ & $\begin{array}{l}\text { Combination of manual and } \\
\text { herbicidal treatment }\end{array}$ & Plant & $\mathrm{F}$ & 4 & 3 & 3 & 4 & 3 & 5 & 3 & 3 & $\mathrm{H}$ \\
\hline $\begin{array}{l}\text { Alopochen aegyptiacus } \\
\text { (Egyptian Goose) }\end{array}$ & $\begin{array}{l}>5000 \text { individuals over half in } \\
\text { Norfolk others in Thames Basin } \\
\text { and Midlands }\end{array}$ & $\begin{array}{l}\text { Primarily shooting, } \\
\text { supplemented by trapping }\end{array}$ & Bird & $\mathrm{T}$ & 4 & 3 & 2 & 4 & 3 & 4 & 4 & 3 & $\mathrm{M}$ \\
\hline $\begin{array}{l}\text { Ichthyosaura alpestris } \\
\text { (Alpine Newt) }\end{array}$ & $\begin{array}{l}\text { c. } 40 \text { populations, mainly in } \\
\text { garden ponds (lentic) }\end{array}$ & $\begin{array}{l}\text { Intensive capture and } \\
\text { destructive techniques }\end{array}$ & Amp. & $\mathrm{F}$ & 4 & 3 & 2 & 5 & 3 & 4 & 1 & 3 & $\mathrm{M}$ \\
\hline $\begin{array}{l}\text { Podarcis muralis } \\
\text { (Wall Lizard) }\end{array}$ & $\begin{array}{l}\text { Well established in c. } 40 \text { sites } \\
\text { with } 20,000+\text { individuals }\end{array}$ & $\begin{array}{l}\text { Intensive capture and habitat } \\
\text { manipulation }\end{array}$ & Rept. & $\mathrm{T}$ & 5 & 3 & 2 & 4 & 2 & 3 & 2 & 3 & $\mathrm{M}$ \\
\hline $\begin{array}{l}\text { Egeria densa } \\
\text { (Large-flowered } \\
\text { Waterweed) }\end{array}$ & $\begin{array}{l}\text { c. } 95 \text { scattered sites; well } \\
\text { established in half, primarily in } \\
\text { rivers, canals and ponds (lotic) }\end{array}$ & $\begin{array}{l}\text { Repeated use of dyes and } \\
\text { manual methods }\end{array}$ & Plant & $\mathrm{F}$ & 3 & 2 & 1 & 2 & 3 & 5 & 3 & 3 & $\mathrm{H}$ \\
\hline $\begin{array}{l}\text { Sagittaria latifolia } \\
\text { (Duck Potato) }\end{array}$ & $\begin{array}{l}\text { c. } 40 \text { populations in lentic and } \\
\text { lotic systems, } 50 \% \text { well } \\
\text { established }\end{array}$ & $\begin{array}{l}\text { Combination of manual and } \\
\text { herbicidal treatment }\end{array}$ & Plant & $\mathrm{F}$ & 3 & 3 & 2 & 2 & 2 & 3 & 3 & 2 & $\mathrm{M}$ \\
\hline
\end{tabular}


Table 3 continued

\begin{tabular}{|c|c|c|c|c|c|c|c|c|c|c|c|c|c|}
\hline \multirow[b]{2}{*}{ Species } & \multirow[b]{2}{*}{$\begin{array}{l}\text { Invasion scenario } \\
\text { (brief summary) }\end{array}$} & \multirow[b]{2}{*}{$\begin{array}{l}\text { Eradication strategy } \\
\text { (brief summary) }\end{array}$} & \multirow[b]{2}{*}{ 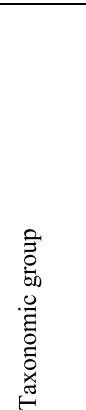 } & \multirow[b]{2}{*}{ 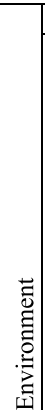 } & \multicolumn{9}{|c|}{ Risk management } \\
\hline & & & & & 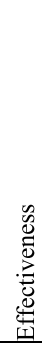 & 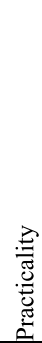 & $\begin{array}{l}\dot{8} \\
\dot{8}\end{array}$ & $\begin{array}{l}\tilde{\mathscr{g}} \\
\tilde{\Xi} \\
\underline{\Xi}\end{array}$ & 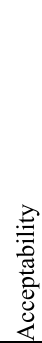 & 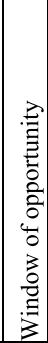 & 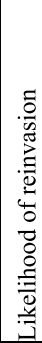 & 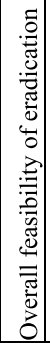 & $\begin{array}{c} \\
0 \\
0 \\
0 \\
0 \\
0 \\
0 \\
0 \\
0 \\
0 \\
0 \\
0\end{array}$ \\
\hline $\begin{array}{l}\text { Orconectes virilis } \\
\text { (Virile Crayfish) }\end{array}$ & $\begin{array}{l}1 \text { population in the River Lee } \\
\text { catchment (lotic) }\end{array}$ & $\begin{array}{l}\text { High density trapping and male } \\
\text { sterilisation }\end{array}$ & Invert. & $\mathrm{F}$ & 2 & 2 & 2 & 2 & 1 & 4 & 3 & 2 & $\mathrm{M}$ \\
\hline $\begin{array}{l}\text { Procambarus clarkii } \\
\text { (Red-swamp Crayfish) }\end{array}$ & $\begin{array}{l}\text { Populations in ponds, single } \\
\text { river and canal (lentic and lotic) }\end{array}$ & $\begin{array}{l}\text { Isolate waterbodies and treat } \\
\text { with biocides }\end{array}$ & Invert. & $\mathrm{F}$ & 2 & 2 & 2 & 2 & 1 & 3 & 2 & 2 & $\mathrm{M}$ \\
\hline $\begin{array}{l}\text { Lysichiton americanus } \\
\text { (American Skunk-cabbage) }\end{array}$ & $\begin{array}{l}\text { c. } 800 \text { populations across } \mathrm{GB}, \\
50 \% \text { well established }\end{array}$ & $\begin{array}{l}\text { Combination of manual and } \\
\text { herbicidal treatment }\end{array}$ & Plant & $\mathrm{T}$ & 4 & 2 & 1 & 3 & 2 & 1 & 1 & 2 & $\mathrm{M}$ \\
\hline $\begin{array}{l}\text { Hemigrapsus sanguineus } \\
\text { (Asian Shore Crab) }\end{array}$ & $\begin{array}{l}2 \text { locations, potentially } \\
\text { undetected elsewhere }\end{array}$ & $\begin{array}{l}\text { Trapping supplemented by } \\
\text { scuba collection }\end{array}$ & Invert. & $\mathrm{M}$ & 1 & 2 & 2 & 4 & 4 & 3 & 2 & 1 & $\mathrm{H}$ \\
\hline $\begin{array}{l}\text { Hemigrapsus takanoi } \\
\text { (Brush-clawed shore crab) }\end{array}$ & $\begin{array}{l}2 \text { locations, potentially } \\
\text { undetected elsewhere }\end{array}$ & $\begin{array}{l}\text { Trapping supplemented by } \\
\text { scuba collection }\end{array}$ & Invert. & $\mathrm{M}$ & 1 & 2 & 2 & 4 & 4 & 3 & 2 & 1 & $\mathrm{H}$ \\
\hline $\begin{array}{l}\text { Dreissena bugensis } \\
\text { (Quagga Mussel) }\end{array}$ & $\begin{array}{l}\text { Single catchment, one tributary } \\
\text { and main river }\end{array}$ & $\begin{array}{l}\text { Biocides with damning / draw } \\
\text { down where possible }\end{array}$ & Invert. & $\mathrm{F}$ & 2 & 1 & 1 & 1 & 1 & 2 & 1 & 1 & $\mathrm{H}$ \\
\hline
\end{tabular}

(b) Horizon species (not yet established in GB). Note that these species are not yet established in GB; the scenarios listed were considered the most likely invasion scenarios at the point of detection should the species invade in the future

\begin{tabular}{|c|c|c|c|c|c|c|c|c|c|c|c|c|c|}
\hline $\begin{array}{l}\text { Nassella neesiana } \\
\text { (Chilean Needle Grass) }\end{array}$ & 2 small populations & $\begin{array}{l}\text { Herbicide and follow up } \\
\text { monitoring }\end{array}$ & Plant & $\mathrm{T}$ & 5 & 5 & 5 & 4 & 5 & 4 & 4 & 5 & M \\
\hline $\begin{array}{l}\text { Corvus splendens } \\
\text { (House Crow) }\end{array}$ & 1 population $<10$ birds & $\begin{array}{l}\text { Shooting, supplemented by egg } \\
\text { oiling }\end{array}$ & Bird & $\mathrm{T}$ & 5 & 4 & 5 & 4 & 4 & 4 & 4 & 5 & $\mathrm{H}$ \\
\hline $\begin{array}{l}\text { Tamias sibiricus } \\
\text { (Siberian Chipmunk) }\end{array}$ & $\begin{array}{l}1 \text { dispersed population, } 10 \mathrm{~s} \text { of } \\
\text { individuals }\end{array}$ & $\begin{array}{l}\text { Trapping supplemented by } \\
\text { shooting }\end{array}$ & Mam. & $\mathrm{T}$ & 5 & 5 & 5 & 5 & 5 & 3 & 2 & 5 & $\mathrm{H}$ \\
\hline $\begin{array}{l}\text { Procyon lotor } \\
\text { (Raccoon) }\end{array}$ & 1 population $<10$ individuals & Trapping & Mam. & $\mathrm{T}$ & 5 & 5 & 5 & 5 & 4 & 3 & 2 & 5 & $\mathrm{H}$ \\
\hline $\begin{array}{l}\text { Threskiornis aethiopicus } \\
\text { (Sacred Ibis) }\end{array}$ & 1 population $(<10$ birds $)$ & $\begin{array}{l}\text { Shooting, supplemented by } \\
\text { trapping }\end{array}$ & Bird & $\mathrm{T}$ & 5 & 4 & 5 & 4 & 3 & 3 & 2 & 5 & $\mathrm{H}$ \\
\hline $\begin{array}{l}\text { Microstegium vimineum } \\
\text { (Japanese Stiltgrass) }\end{array}$ & 1 population & Hand pulling and herbicide & Plant & $\mathrm{T}$ & 4 & 4 & 5 & 4 & 4 & 4 & 4 & 4 & $\mathrm{M}$ \\
\hline $\begin{array}{l}\text { Ocenebra inornata } \\
\text { (Japanese Sting Winkle) }\end{array}$ & $\begin{array}{l}\text { population in a single oyster } \\
\text { rm }\end{array}$ & $\begin{array}{l}\text { Mechanical removal targeting } \\
\text { eggs }\end{array}$ & Invert. & M & 4 & 4 & 4 & 4 & 4 & 5 & 3 & 4 & $\mathrm{~L}$ \\
\hline $\begin{array}{l}\text { Nyctereutes procyonoides } \\
\text { (Raccoon Dog) }\end{array}$ & 1 population & Trapping & Mam. & $\mathrm{T}$ & 4 & 4 & 4 & 5 & 4 & 3 & 2 & 4 & $\mathrm{M}$ \\
\hline $\begin{array}{l}\text { Vespa velutina } \\
\text { (Asian Hornet) }\end{array}$ & 3 nests, some high in trees & Pesticide and nest destruction & Invert. & $\mathrm{T}$ & 4 & 3 & 3 & 5 & 5 & 2 & 2 & 3 & $\mathrm{~L}$ \\
\hline $\begin{array}{l}\text { Homarus americanus } \\
\text { (American Lobster) }\end{array}$ & $\begin{array}{l}2 \text { well established populations } \\
\text { establish off south coast }\end{array}$ & $\begin{array}{l}\text { Trapping supplemented by scuba } \\
\text { collection and possibly male } \\
\text { sterilisation }\end{array}$ & Invert. & $\mathrm{M}$ & 2 & 3 & 2 & 5 & 4 & 4 & 3 & 2 & $\mathrm{M}$ \\
\hline $\begin{array}{l}\text { Linepithema humile } \\
\text { (Argentine Ant) }\end{array}$ & 1 population in garden & $\begin{array}{l}\text { Application of insecticidal bait } \\
\text { and post treatment monitoring }\end{array}$ & Invert. & $\mathrm{T}$ & 3 & 3 & 5 & 4 & 3 & 3 & 2 & 2 & $\mathrm{M}$ \\
\hline $\begin{array}{l}\text { Rapana venosa } \\
\text { (Rapa Whelk) }\end{array}$ & $\begin{array}{l}\text { Multiple populations in } \\
\text { estuary (near oyster beds), } \\
\text { partially accessible }\end{array}$ & $\begin{array}{l}\text { Physical removal by whelk } \\
\text { fishermen and volunteers }\end{array}$ & Invert. & M & 2 & 3 & 3 & 5 & 4 & 3 & 2 & 2 & $\mathrm{H}$ \\
\hline $\begin{array}{l}\text { Proterorhinus marmoratus } \\
\text { (Tubenose Goby) }\end{array}$ & $\begin{array}{l}1 \text { well established population } \\
\text { in a salt and freshwater } \\
\text { system }\end{array}$ & Rotenone based piscicides & Fish & $\mathrm{F}$ & 2 & 2 & 2 & 2 & 3 & 2 & 2 & 2 & $\mathrm{H}$ \\
\hline $\begin{array}{l}\text { Neogobius melanostomus } \\
\text { (Round Goby) }\end{array}$ & $\begin{array}{l}1 \text { well established population } \\
\text { in a salt and freshwater } \\
\text { system }\end{array}$ & Rotenone based piscicides & Fish & $\mathrm{F}$ & 2 & 2 & 2 & 2 & 3 & 2 & 2 & 2 & $\mathrm{H}$ \\
\hline
\end{tabular}


Table 3 continued

\begin{tabular}{|c|c|c|c|c|c|c|c|c|c|c|c|c|c|}
\hline \multirow[b]{2}{*}{ Species } & \multirow[b]{2}{*}{$\begin{array}{l}\text { Invasion scenario } \\
\text { (brief summary) }\end{array}$} & \multirow[b]{2}{*}{$\begin{array}{l}\text { Eradication strategy } \\
\text { (brief summary) }\end{array}$} & \multirow[b]{2}{*}{ 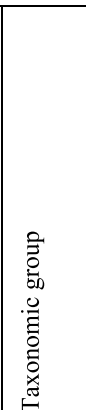 } & \multirow[b]{2}{*}{ 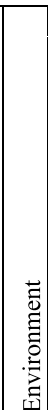 } & \multicolumn{9}{|c|}{ Risk management } \\
\hline & & & & & 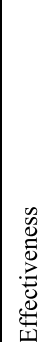 & 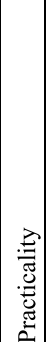 & $\overrightarrow{0}$ & 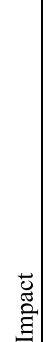 & 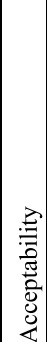 & 疍 & 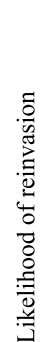 & 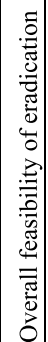 & 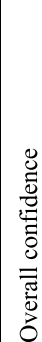 \\
\hline $\begin{array}{l}\text { Corbicula fluminalis } \\
\text { (Asian Clam) }\end{array}$ & $\begin{array}{l}1 \text { well established population } \\
\text { in a freshwater system (e.g. } \\
\text { Norfolk Broads) }\end{array}$ & Application of potash & Invert. & $\mathrm{F}$ & 2 & 2 & 2 & 3 & 3 & 2 & 1 & 2 & $\mathrm{M}$ \\
\hline $\begin{array}{l}\text { Celtodoryx ciocalyptoides } \\
\text { (A sponge) }\end{array}$ & $\begin{array}{l}\text { Well established populations } \\
\text { in multiple oyster farms }\end{array}$ & $\begin{array}{l}\text { Hand removal and chemical } \\
\text { treatment }\end{array}$ & Invert. & M & 2 & 1 & 3 & 5 & 4 & 4 & 3 & 1 & $\mathrm{H}$ \\
\hline $\begin{array}{l}\text { Myriophyllum } \\
\text { heterophyllum } \\
\text { (American Water-milfoil) }\end{array}$ & $\begin{array}{l}2 \text { small populations on a canal } \\
\text { (lentic) }\end{array}$ & Physical and chemical methods & Plant & $\mathrm{F}$ & 1 & 2 & 3 & 3 & 3 & 3 & 2 & 1 & $\mathrm{H}$ \\
\hline $\begin{array}{l}\text { Gracilaria vermiculophylla } \\
\text { (Rough agar weed) }\end{array}$ & $\begin{array}{l}\text { Well established populations } \\
\text { discovered in multiple oyster } \\
\text { farms }\end{array}$ & Mechanical removal & Plant & $\mathrm{M}$ & 1 & 1 & 3 & 4 & 3 & 3 & 1 & 1 & $\mathrm{H}$ \\
\hline $\begin{array}{l}\text { Echinogammarus ischnus } \\
\text { (Bald urchin shrimp) }\end{array}$ & $\begin{array}{l}1 \text { population in a freshwater } \\
\text { system, not widely dispersed }\end{array}$ & Use of biocides & Invert. & $\mathrm{F}$ & 2 & 1 & 3 & 1 & 1 & 2 & 2 & 1 & $\mathrm{H}$ \\
\hline $\begin{array}{l}\text { Echinogammarus trichiatus } \\
\text { (Curly haired urchin } \\
\text { shrimp) }\end{array}$ & $\begin{array}{l}1 \text { population in a freshwater } \\
\text { system, not widely dispersed }\end{array}$ & Use of biocides & Invert. & $\mathrm{F}$ & 2 & 1 & 3 & 1 & 1 & 2 & 2 & 1 & $\mathrm{H}$ \\
\hline $\begin{array}{l}\text { Mnemiopsis leidyi } \\
\text { (American Comb Jelly) }\end{array}$ & $\begin{array}{l}\text { Well established population } \\
\text { detected off south-east coast }\end{array}$ & $\begin{array}{l}\text { No effective eradication } \\
\text { methods }\end{array}$ & Invert. & $\mathrm{M}$ & 1 & 1 & 1 & 1 & 1 & 1 & 1 & 1 & $\mathrm{H}$ \\
\hline
\end{tabular}

Species are grouped according to the overall feasibility of eradication from Great Britain. Colours and numbers reflect response scores (see Table 1) with overall feasibility of eradication scored from 1 (very low) to 5 (very high). Confidence, rated L (low), M (medium) and $\mathrm{H}$ (high), was recorded for all response scores, but for simplicity is only provided here for overall score. Broad taxonomic group (Invert. invertebrate, Amp. amphibian, Rept. reptile, Mam. mammal) is provided as well as main environment in which the species occurs ( $M$ marine, $F$ freshwater, $T$ terrestrial)

workshop. The first phase of the workshop commenced in plenary with a presentation and discussion around the criteria and scoring approach, followed by presentations of initial scores by group leaders with all workshop participants invited to discuss scores and provide challenge. The aim of this exercise was to provide an opportunity to resolve any ambiguity about the process, encourage consistency in scoring between expert groups and review scores. After initial scores had been considered by all participants the expert groups were reformed to discuss and agree alteration of scores if necessary.

In the second phase of the workshop the facilitators presented the refined scores for all species in plenary to all participants. Participants were asked to review and modify these scores if necessary. By the end of this second phase, all response and confidence scores were agreed by the consensus of all participants.
Analysis

We examined individual relationships between overall score and the sub-scores for the seven detailed risk management questions using polychoric correlations as the scores were ordinal from 1 to 5 (see Table 1). We carried out a factor analysis of the individual scores and examined the relationship between scores for all 41 species using non-metric multi-dimensional scaling (nMDS). Changes to all confidence scores (i.e. for each of the seven risk management questions and the overall score) were assessed from the initial scores to final scores at the end of the second phase of the workshop.

To indicate priorities for eradication a matrix was used to compare overall risk management scores with existing risk assessment scores (Fig. 3). Within this matrix, species that scored the highest risk and highest 
feasibility of eradication were given greatest priority, while species that scored less on either axis were lower priority. A symmetric relationship between risk assessment and risk management scores was assumed, such that a species of 'high' risk and 'medium' feasibility of eradication received the same priority as a species of 'medium' risk and 'high' feasibility of eradication. Risk assessment scores were derived from published data, with the GB Non-native Risk Assessment scheme (Baker et al. 2008) providing data for established species (published at www.nonnativespecies. org) and Roy et al. (2014c) providing data for horizon species. These two schemes differ in that the GB Nonnative Risk Assessment scheme provides an overall score of high, medium or low risk; whereas horizon species were all assessed as high risk by Roy et al. (2014c) and were then further sub-divided into the top 10 , top 20 and top 30 threats. This difference is reflected in the two prioritisation matrices produced.

\section{Results}

Risk management scores for all 41 established and horizon species were agreed by consensus (Table 3a, b). There was a broad spread of scores for overall feasibility of eradication, with $13-25 \%$ of the species falling into each of the five possible response categories (i.e. 1-very low to 5-very high).

The score for overall feasibility of eradication was most strongly correlated with the risk management components Practicality (polychoric correlation \pm standard error $0.97 \pm 0.02)$, Effectiveness $(0.93 \pm 0.03)$ and to a lesser extent Cost $(0.64 \pm 0.1)$. There was no significant correlation between overall feasibility of eradication and Impact, Acceptability, Window of opportunity or Likelihood of reinvasion (Fig. 1a).

The data were too sparse to predict overall feasibility of eradication by modelling sub-scores (i.e. scores from each of the seven key risk management questions). However, accounting for inter-correlations through a factor analysis and nMDS showed the overall assessment of feasibility of eradication broadly relates to the underlying sub-scores (Fig. 1b). Coordinate one of the nMDS correlated with overall feasibility of eradication, with minimal overlap of overall scores except between scores 1 and 2 (i.e. 'very low' overall feasibility and 'low' feasibility of eradication respectively).
Both response and confidence scores were refined during the workshop, with $26 \%$ of response scores and $58 \%$ of confidence scores modified during the first phase, and $5 \%$ of response and $2 \%$ of confidence scores further modified during the second phase. Confidence increased from the initial scores (proportion of all confidence scores: low $=13 \%$, medium $=87 \%$, high $=0 \%$ ) to the final scores at the end of the second phase (proportion of all confidence scores: low $=8 \%$, medium $=39 \%$, high $=52 \%$ ). A similar number of response scores increased as decreased. Changes in the response and confidence scores for the seven key risk management questions tended to result in similar changes to the scores for overall feasibility of eradication.

We found differences in scores for overall feasibility of eradication between environments $\left(\chi^{2}=23.73\right.$, $d f=8, \mathrm{p}=<0.01$ ), with terrestrial species generally scoring 'very high', 'high' or 'medium' feasibility; freshwater species scoring 'medium' or lower feasibility; and marine species scoring 'low' or 'very low' feasibility (Fig. 2). We did not detect differences between taxonomic groups, although the representative sample size for each group was low.

The scores for overall feasibility of eradication were combined with overall risk assessment scores to produce separate prioritisation matrices for established and horizon species (Fig. 3a, b). Overall, 12 of the 41 species assessed scored 'high', 'very high' or 'highest' priority for eradication. Established species were divided into four groups of differing priority ranging from 'very high' to 'low' priority with each group comprising 2-8 species and six species scoring 'high' or 'very high' priority. Horizon species were divided into seven groups of differing priority ranging from 'highest' to 'lowest' with each group comprising 1-5 species and six species scoring 'high', 'very high' or 'highest' priority. There was no positive correlation demonstrated between risk assessment and risk management (Fig. 3a, b) and the combination of the two provided information not apparent when considering either risk assessment or risk management in isolation.

\section{Discussion}

We demonstrate that the NNRM is a practical scheme that can be used to assess a wide range of taxa from different environments and directly 
Fig. 1 Factor analysis and non-metric multi-

dimensional scaling

(nMDS) showing

relationship between overall feasibility of eradication and subscores (i.e.

Effectiveness, Practicality, Cost, Impact, Acceptability, Window of opportunity and Likelihood of reinvasion). a Factor analysis showing correlation between risk management sub-scores. The contribution of each factor to each dimension is represented by the length and colour of arrows and overall explain $72.2 \%$ of the variance in the data. Parallel arrows indicate correlation of factors. b Non-metric multi-dimensional scaling of sub-scores with each species coloured by overall feasibility of eradication score. The shaded ellipses are a visual aid centred around the mean showing variation (scaled shape and size of the ellipse) of overall score. Coordinate 1 correlated well with overall feasibility of eradication (1-5)

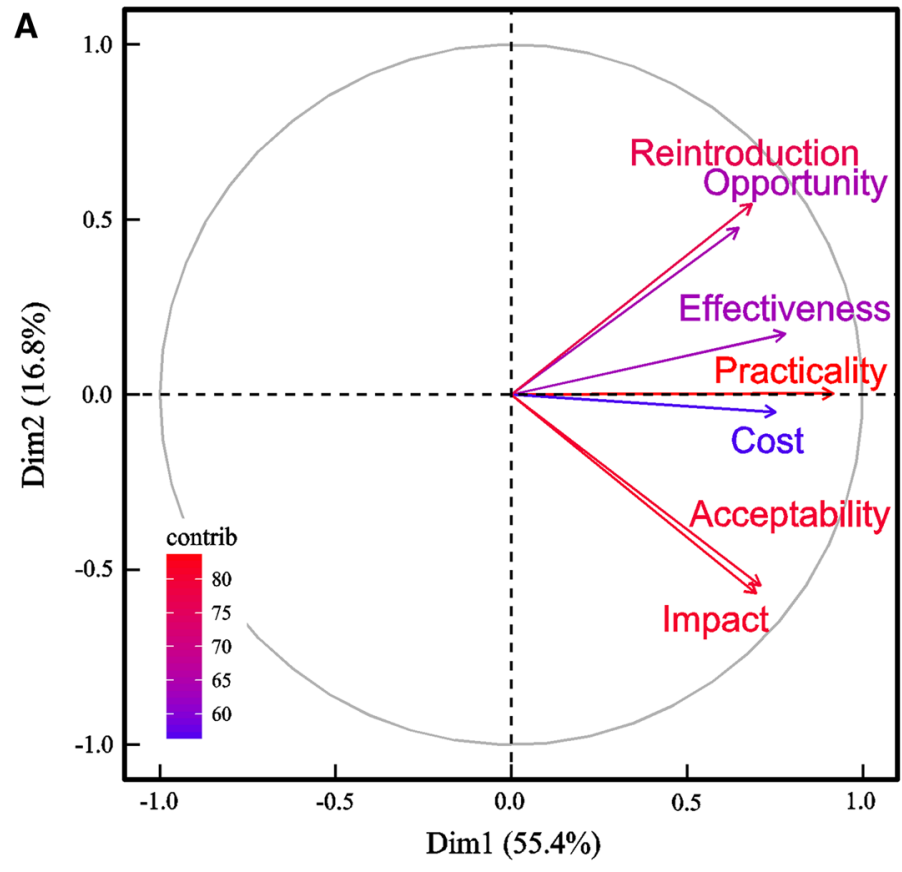

B

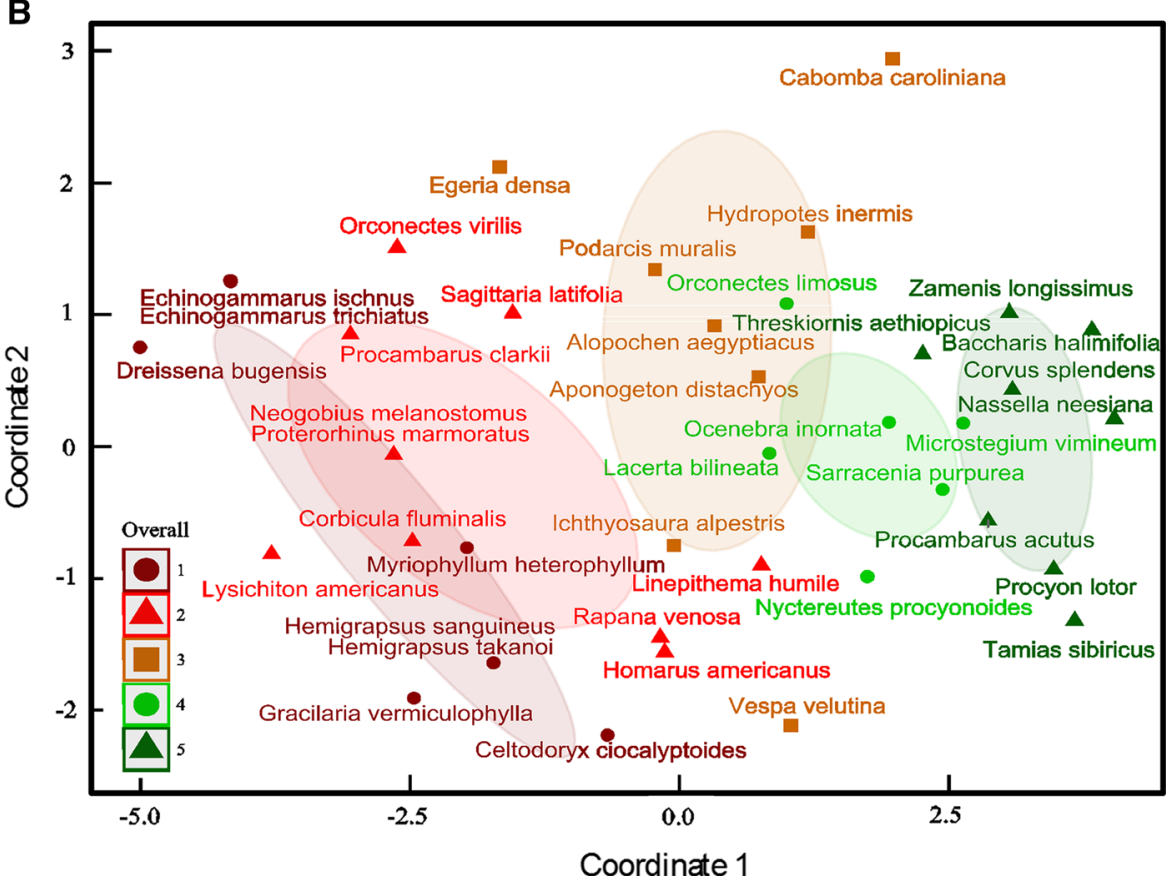

compare them according to the overall feasibility of eradication. It complies with international standards for risk management (FAO 1995; OIE 2015) and good practice for non-native species prioritisation (summarised by Heikkilä 2011) and is compatible with existing risk assessment schemes (Baker et al.
2008, 2012). In conjunction with risk assessment scores, the NNRM can be used to indicate priorities for eradication of existing and future invasive non-native species. With increasing legislative requirements to manage INNS, decision makers require a rapidly applied, transparent and defendable process by which 


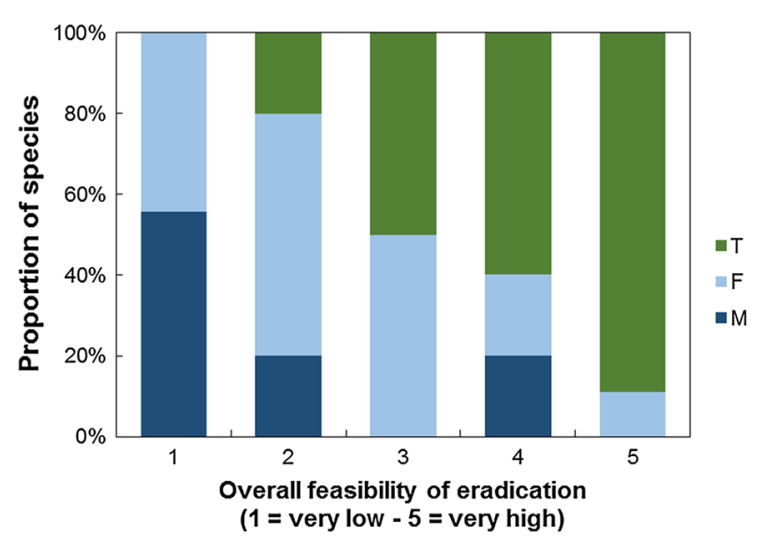

Fig. 2 Overall feasibility of eradicating species based on environment: $T$ terrestrial, $F$ freshwater, $M$ marine. Overall feasibility of eradication is shown as a proportion of the species assessed

eradication actions can be prioritised for established species, and contingency plans developed for horizon species (Early et al. 2016). Not only does the NNRM facilitate risk based policy making in relation to the eradication of INNS, but also indicates other potential management actions where feasibility of eradication is low (e.g. targeted measures to prevent introduction or containment measures) as well as providing broad estimates of cost allowing for more effective budget management. While applied here to GB, the scheme can be applied to any defined area.

We found that expert scoring, based on predefined semi-quantitative scales, coupled with consensus building methods, was a practical way of eliciting robust standardised risk management scores across taxa and environment, even where data were incomplete or uncertain. It was important to reduce the potential impact of subjectivity and bias, which we did following the approach of Roy et al. (2014c). This also provided additional benefits in the exchange of knowledge between a diverse group of experts that do not regularly engage, leading to the challenge of preconceptions about management in some cases. While we found this approach was effective and practical, good practice in the use of experts continues to develop and should be considered in further applications of the scheme. This could include providing additional training steps for scorers using known data, using and evaluating scoring intervals and using multiple experts to independently score species before and after discussions (Hanea et al. 2016; Martin et al. 2012; Sutherland and Burgman 2015).
A key aim of the consensus workshop was to provide an opportunity to refine scores based on knowledge exchange between experienced INNS managers and to ensure participants had a clear and consistent understanding of the guidance. This resulted in a number of changes to scores throughout the workshop, the majority of which were made during the first phase, which was the first opportunity participants had to make refinements following clarification of the guidance and extensive discussions within and between expert groups. The decrease in the number of changes made to assessment scores between the first and second phase of the workshop demonstrates consensus amongst the experts being achieved. Confidence scores increased throughout the workshop with the majority of scores increasing by one degree (i.e. from medium to high) during the first phase. While expert judgement often suffers from overconfidence (Hulme 2012; Morgan 2014), this suggests that individual assessors were initially cautious when providing draft scores, but confidence improved with clarification of the guidance and the benefit of collective experience. The increase in confidence was a consistent pattern across all expert groups, suggesting it was not driven by one or two individuals convincing others.

The strong correlation between overall score and Practicality, Effectiveness and to a lesser degree Cost indicates that these components are the most consistent factors when considering overall feasibility of eradication. The lack of correlation with Likelihood of reinvasion and Window of opportunity indicates that these components carry less weight in determining the overall feasibility of eradication; however, they do provide important additional information that may influence resource allocation and the timing of management. For example, while the purple pitcher-plant (Sarracenia purpuria) received a high score for overall feasibility of eradication, it received only a medium score for Likelihood of reinvasion, suggesting that if eradication were attempted, effort would be required to prevent reinvasion through further deliberate planting in the wild by carnivorous plant enthusiasts. Impact and Acceptability also did not correlate strongly with overall score, but did have a pronounced impact on the overall feasibility of eradication for some species. For example, while Carolina fanwort (Cabomba caroliniana) occurs in only one location in $\mathrm{GB}$, the feasibility of its 


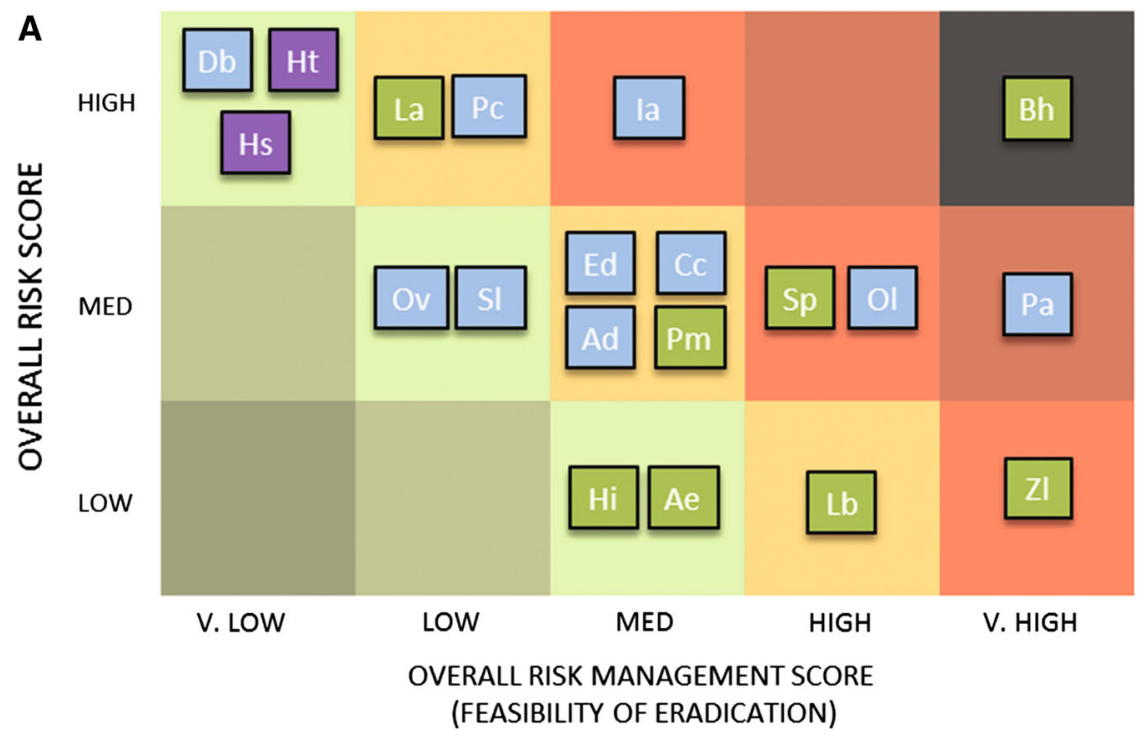

\begin{tabular}{|l|l|}
\hline Priority & Species \\
\hline Highest & Baccharis halimifolia \\
\hline Very high & Procambarus acutus \\
\hline High & Zamenis longissimus \\
\cline { 2 - 2 } & Sarracenia purpurea \\
\cline { 2 - 2 } & Orconectes limosus \\
\cline { 2 - 2 } & Ichthyosauraalpestris \\
\hline Moderate & Lacerta bilineata \\
\cline { 2 - 2 } & Egeria densa \\
\cline { 2 - 2 } & Cabomba caroliniana \\
\cline { 2 - 2 } & Aponogeton distachyos \\
\cline { 2 - 2 } & Podarcis muralis \\
\cline { 2 - 2 } & Lysichiton americanus \\
\cline { 2 - 2 } & Procambarus clarkii \\
\hline \multirow{5}{*}{ Low } & Hydropotes inermis \\
\cline { 2 - 2 } & Alopochen aegyptiacus \\
\cline { 2 - 2 } & Orconectes virilis \\
\cline { 2 - 2 } & Sagittaria latifolia \\
\cline { 2 - 2 } & Dreissena bugensis \\
\cline { 2 - 2 } & Hemigrapsus sanguineus \\
\cline { 2 - 2 } & Hemigrapsus takanoi \\
\hline
\end{tabular}

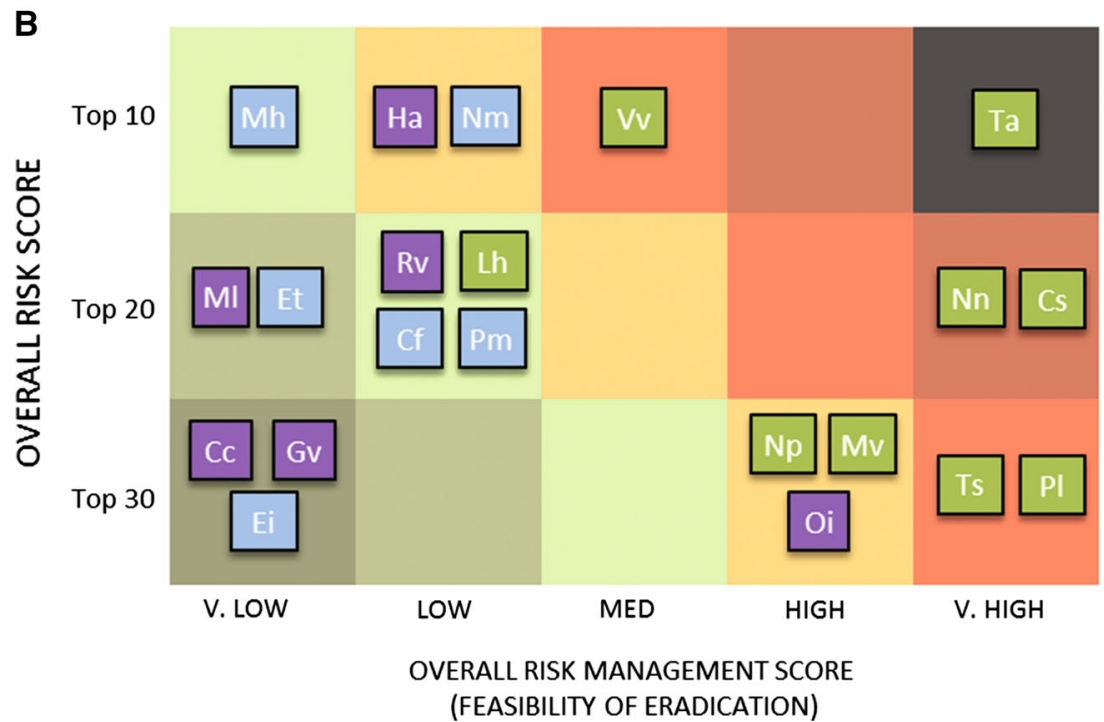

\begin{tabular}{|l|l|}
\hline Priority & Species \\
\hline Highest & Threskiornis aethiopicus \\
\hline Very high & Nassella neesiana \\
\cline { 2 - 2 } & Corvus splendens \\
\hline \multirow{4}{*}{ High } & Procyon lotor \\
\cline { 2 - 2 } & Tamias sibiricus \\
\cline { 2 - 2 } & Vespa velutina \\
\hline \multirow{5}{*}{ Medium } & Nyctereutes procyonoides \\
\cline { 2 - 2 } & Microstegium vimineum \\
\cline { 2 - 2 } & Ocenebra inornata \\
\cline { 2 - 2 } & Homarus americanus \\
\cline { 2 - 2 } & Neogobius melanostomus \\
\hline \multirow{5}{*}{ Low } & Rapana venosa \\
\cline { 2 - 2 } & Linepithema humile \\
\cline { 2 - 2 } & Corbicula fluminalis \\
\cline { 2 - 2 } & Proterorhinus marmoratus \\
\cline { 2 - 2 } & Myriophyllum heterophyllum \\
\hline Very low & Mnemiopsis leidyi \\
\cline { 2 - 2 } & Echinogammarus trichiatus \\
\hline \multirow{5}{*}{ Lowest } & Celtodoryx ciocalyptoides \\
\cline { 2 - 2 } & Gracilaria vermiculophylla \\
\cline { 2 - 2 } & Echinogammarus ischnus \\
\hline
\end{tabular}

Fig. 3 Using overall risk management and risk assessment scores to indicate priorities for eradication of a established species and $\mathbf{b}$ horizon species. The background colour of the matrix indicates priority (from green $=$ lowest, to black $=$ highest). Initials indicate the position of each species with coloured box representing environment (purple $=$ marine, blue $=$ freshwater, green $=$ terrestrial). Where multiple species occur in one cell they have equal priority and are in no particular order. The accompaning tables show species lists in

eradication was substantially reduced by high levels of impact and low levels of acceptability associated with repeated mechanical control (and potential dredging) where it occurs in an ecologically sensitive Site of Special Scientific Interest. priority order. a Prioritisation matrix for eradicating species already established in GB. Risk assessment scores derived from published risk assessments (available at www.nonnativespecies. org). b Prioritisation matrix for eradication of horizon species based on most likely scenario of invasion in GB. All horizon species were scored as high risk and further grouped into the top 10, top 20 and top 30 threats (i.e. upper 10/30; mid 10/30; and lower 10/30) (Roy et al. 2014c)

We looked for systematic differences in feasibility of eradication across species to explain potential drivers that could further inform prioritisation or management. There was a strong relationship between overall feasibility of eradication and environment, 
with terrestrial species receiving significantly higher scores than aquatics, which broadly reflects the findings of Genovesi (2005), Robertson et al. (2016) and Simberloff (2009). Freshwater species generally received low scores; however, eradication was more likely to be feasible if the species occurred in lentic (still) rather than lotic (flowing) systems. Eradication of marine INNS is notably difficult (Sambrook et al. 2014; Thresher and Kuris 2004) and this group received lowest scores overall. However, eradication in the marine environment may still be feasible when specific conditions are met (e.g. Bax et al. 2002; Culver and Kuris 2000; Wotton et al. 2004), and this is reflected in the result for Japanese sting winkle (Ocenebra inornata). We found no correlation between taxa and overall feasibility of eradication in our data; however, terrestrial vertebrates generally received moderate or higher scores for feasibility of eradication, which reflects experience from GB and elsewhere (Genovesi 2005; Robertson et al. 2016).

When combined with existing risk assessment scores our results demonstrate that the NNRM scheme can be used to prioritise the eradication of large numbers of non-native species across different taxa and environment. We identified 12 out of 41 species that pose a threat to GB as 'high', 'very high' or 'highest' priority for eradication. These priorities are different from those that would result from either risk assessment or risk management alone, indicating that taking both into account provides a more refined approach to prioritisation.

Both established and horizon species can be assessed using the NNRM scheme, allowing for emerging species to be prioritised for eradication and contingency planning to be put in place for new species before they arrive. Six out of the 20 species established in GB were identified as 'high', 'very high' or 'highest' priority for eradication. For these, the extent of establishment appears to be an important factor in determining priorities in some cases (four of the six occurred in one or few small, isolated populations); however, it was not a reliable predictor of priority (three of the seven 'low priority' species were established in two or fewer populations, while two 'high priority' species were comparatively widespread). Of the horizon species, six out of 21 were prioritised as 'high', 'very high' or 'highest' priority for eradication in GB. Prioritising the eradication of these species in advance of an invasion allows for contingency plans to be developed that may increase the efficiency and effectiveness of a response, which is particularly important for species that have a short window of opportunity for eradication, such as the Asian hornet (Vespa velutina). Indeed, such plans are already in place in GB for three of the six priority horizon species identified (published at www. nonnativespecies.org).

Species that are not considered a high priority for eradication may be high priorities for other types of management action. For example, prevention is likely to be a particularly important for high risk species that are not yet established in GB and for which eradication on arrival is unlikely to be feasible. Our results indicate this is likely to be the case for most marine and many freshwater (particularly lotic) INNS, in particular broadleaf watermilfoil (Myriophyllym heterophylum), American lobster (Homarus americanus) and round goby (Neogobius melanostomus). For established species, long term management (e.g. containment or control) may be a priority for those that score high risk and low feasibility of eradication, such as quagga mussel (Dreissena bugensis).

Care should be taken when considering the results of this work in the context of past eradications in GB, as the latter were not the result of a systematic and comprehensive prioritisation process but rather an ad hoc approach largely driven by particular stakeholders or specific political drivers (Sheail 2003). However, some parallels can be drawn as well as exceptions highlighted. Our results indicate that terrestrial and lentic freshwater species are more likely to be priorities for eradication than marine or lotic freshwater species, and this already has been the case in GB where eradications, either complete or underway, have been instigated for terrestrial vertebrates (Himalayan porcupine, Hystrix brachyuran; coypu, Myocastor coypus; muskrat, Ondatra zibethicus (Baker 2010); monk parakeet, Myiopsitta monachus; ruddy duck, Oxyura jamaicensis (Defra 2015; Robertson et al. 2015) and lentic freshwater species (topmough gudegon, Pseudorasbora parva (Britton and Brazier 2006; Britton et al. 2010); fathead minnow, Pimephales promelas; black bullhead, Ameiurus melas; African clawed-frog, Xenopus leavis; American bullfrog, Lithobates catesbeianus; and, water primrose, Luwigia grandiflora (Defra 2015). An important difference between our data and experience from GB to date is that the NNRM scheme indicates terrestrial plants 
could be a high priority for eradication where limited to small populations; however, there are few recorded eradications of these species in GB, or indeed in Europe (Genovesi 2005). We suggest this is because terrestrial plants are often 'sleeper weeds' (Groves 1999) being overlooked at the early stages invasion, with decisions to attempt management taken too late for eradication to be feasible or cost effective. This indicates that greater care should be taken in the future to identify and eradicate potentially invasive terrestrial plants at the earliest opportunity.

This work could be developed in a number of ways. The focus of the scheme is on eradication; however, further tools (or an extension of this scheme) to prioritise species for prevention interventions and long term management are required. Advances have been made in this area in the field of pest and weed risk management (e.g. Auld 2012; FAO 2011; Johnson 2009; Kehlenbeck et al. 2012; Setterfield et al. 2010; Virtue 2010) and similar approaches may be applicable to the broader field of INNS. To aid consistency and repeatability it is important that assessors can clearly define invasion scenarios, eradication strategies and distinguish between the predefined responses used in the semi-quantitative scoring scale. We provide guidance for this purpose; however, further elaborations of the scheme may benefit from refining these further, in particular providing more prescriptive instructions for defining invasion scenarios based on population size and scale; using separate experts to define the scenario than those undertaking the assessment; testing the use of multiple scenarios and eradication strategies for individual species; and, further defining and calibrating the response and confidence scales. A simple assessment of confidence has been presented here, but novel methods have been developed to better utilise and communicate uncertainty that could further enhance the scheme (e.g. Holt et al. 2012).

While applied here at a national level, the scheme is designed for use at any scale from specific sites to continent wide. Indeed, it may be timely to apply the approach across the EU given the requirements for risk management included in the recently adopted Regulation for Invasive Alien Species (Genovesi et al. 2015).

Acknowledgements This work was funded, in part, by the Animal and Plant Health Agency. HER receives support from the Joint Nature Conservation Committee and the Natural
Environment Research Council (via National Capability funding to the Centre for Ecology and Hydrology, Project EC04932). We are grateful to Sonia Vanderhoeven, Johan Näslund and Steve Rushton for comments on the manuscript, as well as all of the experts, risk managers and policy officials that helped throughout the development of the scheme.

Open Access This article is distributed under the terms of the Creative Commons Attribution 4.0 International License (http:// creativecommons.org/licenses/by/4.0/), which permits unrestricted use, distribution, and reproduction in any medium, provided you give appropriate credit to the original author(s) and the source, provide a link to the Creative Commons license, and indicate if changes were made.

\section{References}

Andersen MC, Adams H, Hope B et al (2004) Risk analysis for invasive species: general framework and research needs. Risk Anal 24:893-900

Auld B (2012) An overview of pre-border weed risk assessment and post-border weed risk management protocols. Plant Prot Q 27:105

Baker S (2010) Control and eradication of invasive mammals in Great Britain. Revue scientifique et technique (International Office of Epizootics) 29:311-327

Baker R, Cannon R, Bartlett P et al (2005) Novel strategies for assessing and managing the risks posed by invasive alien species to global crop production and biodiversity. Ann Appl Biol 146:177-191

Baker R, Black R, Copp G et al (2008) The UK risk assessment scheme for all non-native species. In: Rabitsch W, Essl F, Klingenstein F (eds) Biological invasions-from ecology to conservation, vol 7. Neobiota, pp 46-57

Baker RHA, Benninga J, Bremmer J et al (2012) A decisionsupport scheme for mapping endangered areas in pest risk analysis. EPPO Bull 42:65-73

Bax N, Hayes K, Marshall A et al (2002) Man-made marinas as sheltered islands for alien marine organisms: establishment and eradication of an alien invasive marine species. In: Vetch CR, Clout MN (eds) Turning the tide: the eradication of invasive species. IUCN SSC Invasive Species Specialist Group. IUCN [World Conservation Union], Gland and Cambridge, UK, pp 26-39

Bolger F, Rowe G (2015) The aggregation of expert judgment: do good things come to those who weight? Risk Anal 35:5-11

Britton JR, Brazier M (2006) Eradicating the invasive topmouth gudgeon, Pseudorasbora parva, from a recreational fishery in northern England. Fish Manage Ecol 13:329-335

Britton JR, Davies GD, Brazier M (2010) Towards the successful control of the invasive Pseudorasbora parva in the UK. Biol Invasions 12:125

Burgman MA, McBride M, Ashton R et al (2011) Expert status and performance. PLoS ONE 6:e22998

Cacho JO, Spring D, Pheloung P et al (2006) Evaluating the feasibility of eradicating an invasion. Biol Invasions 8:903-917 
Culver CS, Kuris AM (2000) The apparent eradication of a locally established introduced marine pest. Biol Invasions 2:245-253

Cunningham D, Woldendorp G, Burgess M et al (2003) Prioritising sleeper weeds for eradication: Selection of species based on potential impacts on agriculture and feasibility of eradication. Bureau of Rural Sciences, Canberra

D'hondt B, Vanderhoeven S, Roelandt S et al (2015) Harmonia+ and Pandora+: risk screening tools for potentially invasive plants, animals and their pathogens. Biol Invasions 17:1869-1883

Daisie (2009) Handbook of alien species in Europe. Springer, Berlin

Darin GS (2008) Prioritizing weed populations for eradication at a regional level: The California Department of Food and Agriculture's A-rated weeds. University of California, California

Darin GMS, Schoenig S, Barney JN et al (2011) WHIPPET: a novel tool for prioritizing invasive plant populations for regional eradication. J Environ Manag 92:131-139

Defra (2015) The Great Britain invasive non-native species strategy. Defra, London. www.gov.uk/government/ publications

Drolet D, Locke A, Lewis M et al (2014) User-friendly and evidence-based tool to evaluate probability of eradication of aquatic non-indigenous species. $J$ Appl Ecol 51:1050-1056

Early R, Bradley BA, Dukes JS et al (2016) Global threats from invasive alien species in the twenty-first century and national response capacities. Nat Commun 7:12485

Essl F, Nehring S, Klingenstein F et al (2011) Review of risk assessment systems of IAS in Europe and introducing the German-Austrian Black List Information System (GABLIS). J Nat Conserv 19:339-350

EU (2014) Commission regulation (EU) No 1143/2014 of the 22 October 2014 on the prevention and management of the introduction and spread of invasive alien species. Off J Eur Union L 317:35

FAO (1995) Guidelines for pest risk analysis. International standards for phytosanitary measures (ISPM). No. 2. Food and Agriculture Organization of the United Nations, Rome

FAO (2006) Phytosanitary principles for the protection of plants and the application of phytosanitary measures in international trade. International standards for phytosanitary measures (ISPM). No. 1. Food and Agriculture Organization of the United Nations, Rome

FAO (2011) Procedures for post-border weed risk management. Food and Agriculture Organisation of the United Nations, Rome

Firn J, Maggini R, Chadès I et al (2015a) Priority threat management of invasive animals to protect biodiversity under climate change. Glob Change Biol 21:3917-3930

Firn J, Martin TG, Chadès I et al (2015b) Priority threat management of non-native plants to maintain ecosystem integrity across heterogeneous landscapes. J Appl Ecol 52:1135-1144

Genovesi P (2000) Guidelines for eradication of terrestrial vertebrates: a European contribution to the invasive alien species issue. Council of Europe, Strasbourg, tpvs65e-2000

Genovesi P (2005) Eradications of invasive alien species in Europe: a review. In: Capdevila-Argüelles L, Zilletti B (eds) Issues in bioinvasion science. Springer, Netherlands, pp 127-133

Genovesi P (2007) Limits and potentialities of eradication as a tool for addressing biological invasions. In: Nentwig W (ed) Biological invasions. Springer, Berlin, pp 385-402

Genovesi P, Carboneras C, Vila M et al (2015) EU adopts innovative legislation on invasive species: a step towards a global response to biological invasions? Biol Invasions 17:1307-1311

Groves R (1999) Sleeper weeds. In: Proceedings of the 12th Australian weeds conference. Tasmanian Weed Society Devonport, Tasmania, pp 632-636

Hanea A, McBride M, Burgman M et al (2016) Investigate discuss estimate aggregate for structured expert judgement. Int J Forecast 33(1):267-279

Hauser CE, McCarthy MA (2009) Streamlining 'search and destroy': cost-effective surveillance for invasive species management. Ecol Lett 12:683-692

Heikkilä J (2011) A review of risk prioritisation schemes of pathogens, pests and weeds: principles and practices. Agric Food Sci 20:15-28

Hiebert RD, Stubbendieck JL (1993) Handbook for ranking exotic plants for management and control. US Department of the Interior, National Park Service, Natural Resources Publication Office Denver

Holt J, Leach A, Knight J et al (2012) Tools for visualizing and integrating pest risk assessment ratings and uncertainties. EPPO Bull 42:35-41

Hulme PE (2006) Beyond control: wider implications for the management of biological invasions. J Appl Ecol 43:835-847

Hulme PE (2009) Trade, transport and trouble: managing invasive species pathways in an era of globalization. J Appl Ecol 46:10-18

Hulme PE (2012) Weed risk assessment: a way forward or a waste of time? J Appl Ecol 49:10-19

Hulme PE, Pyšek P, Nentwig W et al (2009) Will threat of biological invasions unite the European Union. Science 324:40-41

Johnson S (2009) NSW weed risk management system: instruction book. Department of Industry and Innovation, New South Wales Government

Kehlenbeck H, Cannon R, Breukers A et al (2012) A protocol for analysing the costs and benefits of phytosanitary measures. EPPO Bull 42:81-88

Leung B, Roura-Pascual N, Bacher S et al (2012) TEASIng apart alien species risk assessments: a framework for best practices. Ecol Lett 15:1475-1493

Liu S, Sheppard A, Kriticos D, Cook D (2011) Incorporating uncertainty and social values in managing invasive alien species: a deliberative multi-criteria evaluation approach. Biol Invasions 13(10): 2323

Martin TG, Burgman MA, Fidler F et al (2012) Eliciting expert knowledge in conservation science. Conserv Biol 26:29-38

Mastrandrea MD, Mach KJ, Plattner G-K et al (2011) The IPCC AR5 guidance note on consistent treatment of uncertainties: a common approach across the working groups. Clim Change 108:675-691

McGeoch MA, Genovesi P, Bellingham PJ et al (2016) Prioritizing species, pathways, and sites to achieve conservation targets for biological invasion. Biol Invasions 18:299 
Mehta SV, Haight RG, Homans FR et al (2010) Decision making under risk in invasive species management: risk management theory and applications. General Technical report-pacific northwest research station, USDA Forest Service, pp 445-468

Morgan MG (2014) Use (and abuse) of expert elicitation in support of decision making for public policy. Proc Natl Acad Sci 111:7176-7184

Mumford J, Booy O, Baker R et al (2010) Invasive non-native species risk assessment in Great Britain. Aspects Appl Biol 104:49-54

Nentwig W, Kühnel E, Bacher S (2010) A Generic ImpactScoring System Applied to Alien Mammals in Europe. Conserv Biol 24:302-311

OIE (2015) Terrestrial animal health code. World Organisation for Animal Health, Paris

Pimentel D, Zuniga R, Morrison D (2005) Update on the environmental and economic costs associated with alien-invasive species in the United States. Ecol Econ 52:273-288

Randall JM, Morse LE, Benton N et al (2008) The invasive species assessment protocol: a tool for creating regional and national lists of invasive nonnative plants that negatively impact biodiversity. Invasive Plant Sci Manag $1: 36-49$

Rejmánek M, Pitcairn M (2002) When is eradication of exotic pest plants a realistic goal. In: Vietch C, Clout MN (eds) Turning the tide: the eradication of invasive species. IUCN, The World Conservation Union, pp 249-253

Robertson PA, Adriaens T, Caizergues A et al (2015) Towards the European eradication of the North American ruddy duck. Biol Invasions 17:9

Robertson PA, Adriaens T, Lambin X et al (2016) The largescale removal of mammalian invasive alien species in Northern Europe. Pest Manag Sci 73(2):273-279

Roy HE, Preston CD, Harrower CA et al (2014a) GB non-native species information portal: documenting the arrival of nonnative species in Britain. Biol Invasions 16:2495-2505

Roy HE, Schonrogge K, Dean H et al (2014b) Invasive alien species-framework for the identification of invasive alien species of EU concern. Report to the European Commission, ENV.B.2/ETU/2013/0026

Roy HE, Peyton J, Aldridge DC et al (2014c) Horizon scanning for invasive alien species with the potential to threaten biodiversity in Great Britain. Glob Change Biol 20:3859-3871

Sambrook K, Holt RH, Sharp R et al (2014) Capacity, capability and cross-border challenges associated with marine eradication programmes in Europe: the attempted eradication of an invasive non-native ascidian, Didemnum vexillum in Wales, United Kingdom. Mar Policy 48:51-58

Schmiedel D, Wilhelm E-G, Roth M et al (2016) Evaluation system for management measures of invasive alien species. Biodivers Conserv 25:357-374
Seebens H, Blackburn TM, Dyer EE et al (2017) No saturation in the accumulation of alien species worldwide. Nat Commun 8:14435

Setterfield S, Ferdinands K, Rossiter-Rachor N et al (2010) The Northern Territory's Weed Risk Management System: an application of the national post-border WRM Protocol. Plant Prot Q 25:86

Sheail J (2003) Government and the management of an alien pest species: a British perspective. Landsc Res 28:101-111

Simberloff D (2003) Eradication-preventing invasions at the outset. Weed Sci 51:247-253

Simberloff D (2009) We can eliminate invasions or live with them. Successful management projects. Biol Invasions 11:149-157

Sunley R, Cannon R, Eyre D et al (2012) A decision-support scheme that generates contingency plans and prioritizes action during pest outbreaks. EPPO Bull 42:89-92

Sutherland W, Burgman M (2015) Policy advice: use experts wisely. Nature 526:317-318

Sutherland W, Pullin A, Dolman P, Knight T (2004) The need for evidence-based conservation. Trends Ecol Evol 19(6):305-308

Thresher RE, Kuris AM (2004) Options for managing invasive marine species. Biol Invasions 6:295-300

UNEP (2011) The strategic plan for biodiversity 2011-2020 and the Aichi biodiversity targets. UNEP/CBD/COP/DEC/X/2, 29 October 2010, Nagoya. COP CBD Tenth Meeting. www.cbd.int/decisions/cop/?m=cop-10

Vander Zanden MJ, Hansen GJ, Higgins SN et al (2010) A pound of prevention, plus a pound of cure: early detection and eradication of invasive species in the Laurentian Great Lakes. J Great Lakes Res 36:199-205

Vanderhoeven S, Adriaens T, D'hondt B et al (2015) A sciencebased approach to tackle invasive alien species in Belgium-the role of the ISEIA protocol and the Harmonia information system as decision support tools. Manag Biol Invasions 6(2): 197-208

Vanderhoeven S, Branquart E, Casaer J, D'hondt B, Hulme PE, Shwartz A, Strubbe D, Turbe A, Verreycken H, Adriaens T (2017) Beyond protocols: improving the reliability of expert-based risk analysis underpinning invasive species policies. Biol Invasions. doi:10.1007/s10530-017-1434-0

Verbrugge L, Leuven R, van der Velde G (2010) Evaluation of international risk assessment protocols for exotic species. Department of Environmental Science, Report, p 352

Virtue JG (2010) South Australia's weed risk management system. Plant Prot Q 25:90

Wotton D, O'Brien C, Stuart M et al (2004) Eradication success down under: heat treatment of a sunken trawler to kill the invasive seaweed Undaria pinnatifida. Mar Pollut Bull 49:844-849 